\title{
The prof of the uniqueness of the solution of a mixed problem for a class of partial differentatial equations of even order
}

\author{
by Z. SToJfK (Kraków)
}

In this paper we present the proof of the uniqueness of the solution of a mixed problem in the cylinder for linear partial differential equations of even order; these equations correspond to the Euler-Lagrange equation of given variation problems. The method of the proof is similar to that applied by S. Zaremba, K. Friedrichs and others [1] to hyperbolic equations.

Let us consider the partial differential equation of the $2 n$-th order in the form

$$
\begin{array}{r}
\sum_{h=0}^{n} \sum_{\substack{i_{1}+\ldots+i_{m}=h \\
j_{1}+\ldots+j_{m}=h}}(-1)^{h} \frac{\partial^{h}}{\partial x_{1}^{i_{1}} \ldots \partial x_{m}^{i_{m}}}\left[\begin{array}{c}
a_{i_{1}, \ldots, i_{m}}(X) \frac{\partial^{h} w(X, t)}{\partial x_{1}, \ldots, j_{m}} \\
\partial x_{1}^{j_{1}} \ldots \partial x_{m}^{j_{m}}
\end{array}\right]+ \\
+\mu(X) \frac{\partial^{2} w(X, t)}{\partial t^{2}}=f(X, t)
\end{array}
$$

and the corresponding homogenous equation

$$
\begin{aligned}
\sum_{h=0}^{n} \sum_{\substack{i_{1}+\ldots+i_{m}=h \\
j_{1}+\ldots+j_{m}=h}}(-1)^{h} \frac{\partial^{h}}{\partial x_{1}^{i_{1}} \ldots \partial x_{m}^{i_{m}}}\left[\begin{array}{c}
a_{i_{1}, \ldots, i_{m}}(X) \frac{\partial^{h} w(X, t)}{j_{l, \ldots, j_{m}}} \\
\partial x_{1}^{j_{1}} \ldots \partial x_{m}^{j_{m}}
\end{array}\right]+ \\
+\mu(X) \frac{\partial^{2} w(X, t)}{\partial t^{2}}=0 .
\end{aligned}
$$

Let us suppose that $a_{i_{1}, \ldots, i_{m}}(X)\left(i_{1}+\ldots+i_{m}=h ; j_{1}+\ldots+j_{m}=h\right.$; $h=0,1, \ldots, n)$ belongs to the class $C^{h}$ in $\Omega$ and that the following relations hold:

$$
\begin{aligned}
& \underset{\substack{a_{1}, \ldots, i_{m} \\
j_{1}, \ldots, j_{m}}}{ }(X)=a_{\substack{j_{1}, \ldots, j_{m} \\
i_{1}, \ldots, i_{m}}}(X), \quad X \in \Omega \\
& \left(i_{1}+\ldots+i_{m}=h, j_{1}+\ldots+j_{m}=h, h=1,2, \ldots, n\right)
\end{aligned}
$$

and we assume the continuity of the function $\mu(X)$ in $\Omega$. 
Further, we assume that the domain $\Omega$ is closed, bounded and normal with respect to the system $\left(x_{1}, x_{2}, \ldots, x_{m}\right)$ and its boundary $F \Omega$ is an surface of the class $C_{\sigma}^{1}$ (cf. [1], p. 132).

Theorem 1. If the quadratic form

$$
\sum_{h=0}^{n} \sum_{\substack{i_{1}+\ldots+i_{m}=h \\ j_{1}+\ldots+j_{m}=h}} a_{\substack{i_{1}, \ldots, i_{m} \\ j_{1}, \ldots, i_{m}}}(X) \lambda_{i_{1}, \ldots, i_{m}} \lambda_{j_{1}, \ldots, j_{m}}
$$

is positive semi-definite in $\Omega$, and $\mu(X)>0$ in $\Omega$, then equation (1) within the range of the functions of class $C^{\mathrm{s}}$ with continuous derivatives of $2 n$-th order with regard to spatial variables in the semi-cylinder $\Sigma:\{(X, t) ; X \in \Omega$, $t \geqslant 0\}$ can have at most one solution satisfying the initial conditions

$$
w(X, 0)=\varphi_{0}(X),\left.\quad \frac{\partial w(X, t)}{\partial t}\right|_{t=0}=\varphi_{1}(x) \quad \text { for } \quad X \in \Omega
$$

and the boundary conditions

$$
\begin{array}{r}
\frac{\partial^{h} w(X, t)}{\partial n^{h}}=\psi_{h}(X, t) \quad(h=0,1, \ldots, n-1) \\
\text { for } X \in F \Omega \text { and } t \geqslant 0 .
\end{array}
$$

The truth of this theorem is easily demonstrated by the following

THEOREM 2. If the quadratic form is positive semi-definite in $\Omega$, and $\mu(X)>0$ in $\Omega$, then the only solution of the homogenous equation (2) within the range of the functions of class $C^{3}$ with continuous derivatives of $2 n$-th order with respect to spatial variables in $\Sigma$, fulfilling the initial conditions

$$
w(X, 0)=0,\left.\quad \frac{\partial w(X, t)}{\partial t}\right|_{t=0}=0 \quad \text { for } \quad X \in \Omega
$$

and the boundary conditions

$$
\frac{\partial^{h} w(X, t)}{\partial n^{h}}=0 \quad(h=0,1, \ldots, n-1) \quad \text { for } \quad X \in F \Omega \quad \text { and } \quad t \geqslant 0
$$

is the function identically equal to zero in the semi-cylinder $\Sigma$.

Proof. We multiply both sides of equation (2) by $\partial w / \partial t$ and integrate over the area $D(X \in \Omega, 0 \leqslant t \leqslant T$, where $T$ is an arbitrary number $0<T<\infty)$; we obtain the equality

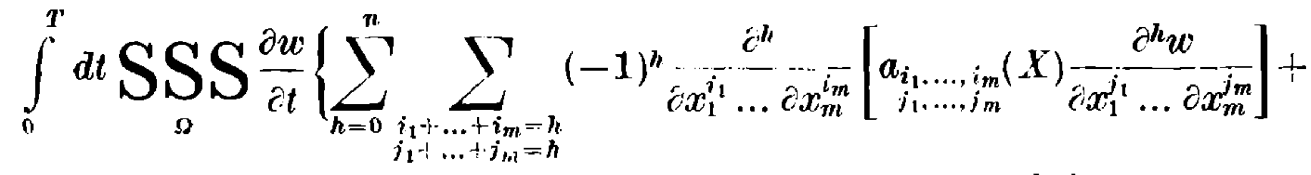

$$
\begin{aligned}
& \left.+\mu(X) \frac{\partial^{2} w}{\partial t^{2}}\right\} d X=0
\end{aligned}
$$


From the formula for integration by parts the $m$-fold integrals [1]

$$
\begin{aligned}
& \underset{\Omega}{\operatorname{SSS}} P(X) \frac{\partial Q(X)}{\partial x_{k}} d X=-\underset{F^{\prime} \Omega}{\operatorname{SS}} P(X) Q(X) \cos \left(n x_{k}\right) d \sigma- \\
&-\operatorname{SSS}_{\Omega} Q(X) \frac{\partial P(X)}{\partial x_{k}} d X \quad(k=1,2, \ldots, m)
\end{aligned}
$$

resulting from Green's theorem, we arrive by induction, for an arbitrary $t \in[0, T]$, at the equality

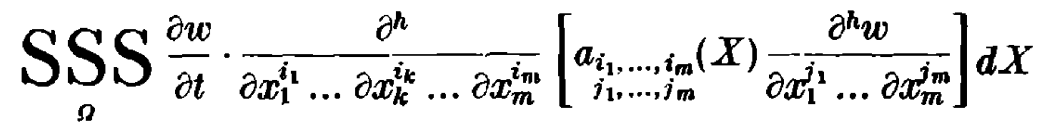

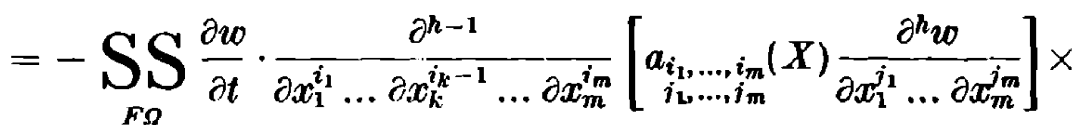

$$
\begin{aligned}
& \times \cos \left(n, x_{k}\right) d \sigma+
\end{aligned}
$$

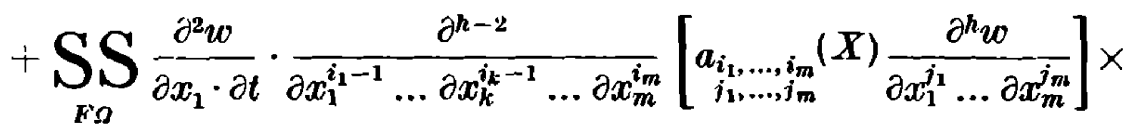

$$
\begin{aligned}
& \times \cos \left(n, x_{1}\right) d \sigma+\ldots+ \\
& +(-1)^{h} \operatorname{SS}_{F \Omega} \frac{\partial^{h} w}{\partial x_{1}^{i_{1}} \ldots \partial x_{k}^{\frac{i_{k}-1}{i_{2}} \ldots \partial x_{m}^{i_{m}} \cdot \partial t}} a_{\substack{i_{1}, \ldots, i_{m} \\
j_{1}, \ldots, j_{m}}}(X) \frac{\partial^{h} w}{\partial x_{1}^{j_{1}} \ldots \partial x_{m}^{j_{m}}} \times \\
& \times \cos \left(n, x_{m}\right) d \sigma+ \\
& +(-1)^{h} \operatorname{SSS}_{\Omega} \frac{\partial^{h+1} w}{\partial x_{1}^{i_{1}} \ldots \partial x_{k}^{i_{k}} \ldots \partial x_{m}^{i_{m}} \cdot \partial t} a_{\substack{i_{1}, \ldots, i_{m} \\
i_{1}, \ldots, j_{m}}}(X) \frac{\partial^{h} w}{\partial x_{1}^{j_{1}} \ldots \partial x_{m}^{j_{m}}} d X \\
& \left(h=1,2, \ldots, n ; i_{k} \geqslant 1 ; i_{1}+\ldots+i_{k}+\ldots+i_{m}=h ;\right. \\
& \left.j_{1}+\ldots+j_{m}=h ; k=1,2, \ldots, m\right) .
\end{aligned}
$$

From the boundary conditions (8) it follows that the derivatives of the functions $w(X, t)$ on the boundary $F \Omega$ equal zero in an arbitrary tangential direction as far as the range $n-1$ for each $t \geqslant 0$, and the derivatives in the dircction of the normal as far as $n-1$ equal zero: hence, for $X \in F \Omega$ and $t \geqslant 0$, we have

$$
\frac{\partial^{h} w}{\partial x_{1}^{i_{1}} \ldots \partial x_{m}^{i_{m}}}=0 \quad\left(h=0,1, \ldots, n-1 ; i_{1}+\ldots+i_{m}=h\right)
$$

and consequently

$$
\begin{aligned}
& \frac{\partial^{h+1} w}{\partial x_{1}^{i_{1}} \ldots \partial x_{m}^{i_{m}} \cdot \partial t}=0 \quad \text { for } \quad X \in F \Omega, \quad t \geqslant 0 \\
& \left(h=0,1, \ldots, n-1 ; i_{1}+\ldots+i_{m}=h\right) \text {. }
\end{aligned}
$$


From (13) it follows that the surface integrals in inequality (11) equal zero, and thus we obtain:

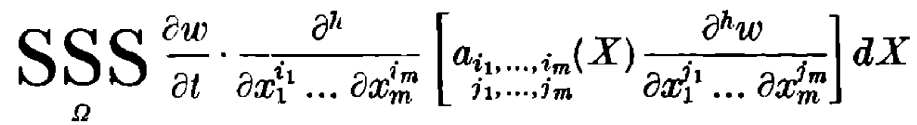

$$
\begin{aligned}
& =(-1)^{h} \operatorname{SSS}_{\boldsymbol{\Omega}} \frac{\partial^{h+1} w}{\partial x_{1}^{i_{1}} \ldots \partial x_{m}^{i_{m}} \cdot \partial t} a_{i_{1}, \ldots, i_{m}}(X) \frac{\partial^{h} w}{\partial x_{1}^{j_{1}} \ldots \partial x_{m}^{j_{m}}} d X \\
& \left(h=1,2, \ldots, n ; i_{1}+\ldots+i_{m}=h ; j_{1}+\ldots+j_{m}=h\right) \text {. }
\end{aligned}
$$

Substituting equality (14) in (9) we arrive at

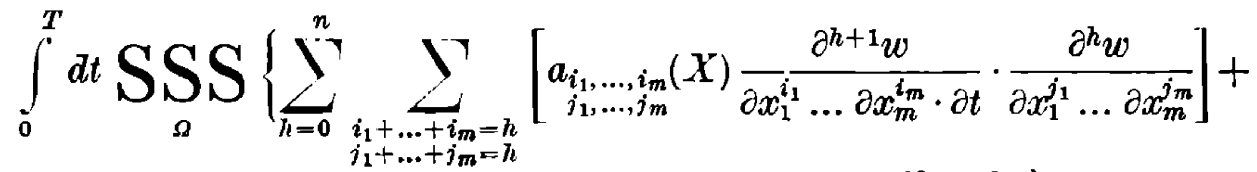

$$
\begin{aligned}
& \left.+\mu(X) \frac{\partial^{2} w}{\partial t^{2}} \cdot \frac{\partial w}{\partial t}\right\} d X=0
\end{aligned}
$$

Considering relations (3), after having integrated the left side of equality (15) with respect to the parameter $t$, we obtain the following equality:

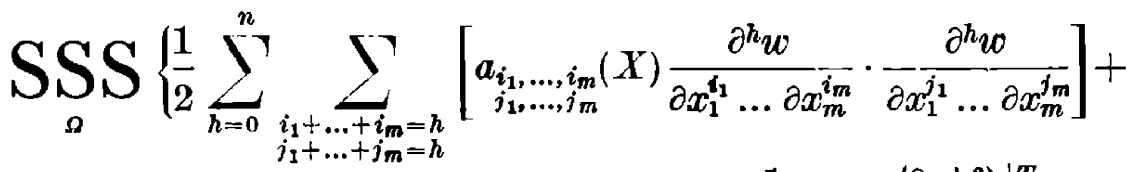

$$
\begin{aligned}
& \left.+\frac{1}{2} \mu(X)\left(\frac{\partial w}{\partial t}\right)^{2}\right\}\left.\right|_{0} ^{T} d X=0 .
\end{aligned}
$$

Since the initial conditions are (7) by $t=0$, the subintegral ex pression equals zero; and since $T$ has been an arbitrary positive number, hence for each $t>0$ the equality

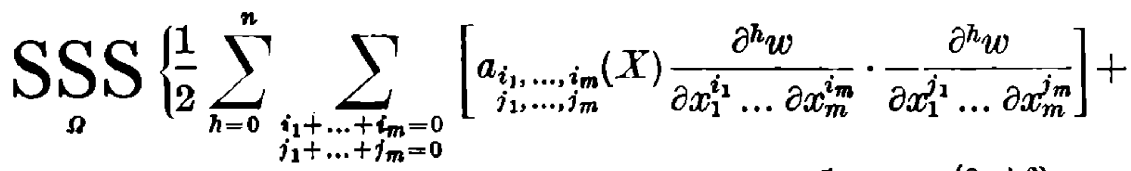

$$
\begin{aligned}
& \left.+\frac{1}{2} \mu(X)\left(\frac{\partial w}{\partial t}\right)^{2}\right\} d X=0
\end{aligned}
$$

is true.

The quadratic form (4) being positive semi-definite in $\Omega, \mu(X)>0$ in $\Omega$ and the continuous derivative of the function $w(X, t)$ being $\partial w / \partial t$, it follows from the equality (17) that in the semi-cylinder $\Sigma$ we have

$$
\frac{\partial w(X, t)}{\partial t} \equiv \mathbf{0}
$$


and that means that the function $w(X, t)$ is independent of the parameter $t$; being continuous in $\Sigma$, this function must also satisfy the homogeneous initial conditions $(7)$; hence $w(X, t) \equiv 0$ in $\Sigma$. Q.e.d.

\section{Reference}

[1] M. Krzyżański, Równania różniczkowe czastkowe rzędu drugiego, Cz. 1., Warszawa 1957.

Reçu par la Rédaction le 3.1.1961 